\title{
TSP Analysis Performed by SEM-EDS to Air Quality Studies
}

\author{
M. Cruz-Campas ${ }^{1}$, R. Ramirez-Leal ${ }^{1 *}$ and N. Lopez-Perez ${ }^{1}$ \\ ${ }^{1}$ Sonora State University, Environmental Engineering. Ley Federal del Trabajo s/n, Col. Apolo \\ Hermosillo, Sonora, Mexico. \\ * Corresponding author: ramz1036@yahoo.com.mx
}

Size and chemical composition of ambient particulates strongly influence on human health, visibility and ecosystem etc. thus it is crucial to investigate the physicochemical characteristics of atmospheric particles and also to evaluate their potential toxicity. Apart from this, Elemental composition of particulate plays an important role in the chemical characteristics of Particulate matters (PM) and provides interesting data, not only for the evaluation of its impact on human health, ecology and environment but also for the identification of specific emission sources and mass is dominates in coarse mode particulate matter in the atmosphere. Surface area may become more essential to ecological impact assessment as recognition of the oxidizing capacity, their interactions with other pollutants such as ozone and their processes [2].

Total suspended particles (TSP) is generally composed of several components including resuspended soil particles, weathered materials, fly ash and vehicle-generated pollutants, and they are ubiquitous environmental pollutants that formed in building construction, combustion of coal wood and atmospheric chemical reaction. But by far, responded road dust, traffic, and other combustion processes are usually deemed to be the principal sources of TSP. Determination of the origin of TSP is often based on their morphological and chemical characteristics. So the study of TSP pollution is important [1].

Knowledge about the size dimensions and the shape and composition of the particles and their individual members are more directly correlated with their effects on public health and the environment; because in the cities the population is exposed to particles that are often combined with other dangerous substances that have been less investigated and therefore little known [3].

TSP particles was collected by high-volume sampler, located in the northwest of the city, using a sampling flow of $1.3 \mathrm{~m} 3 / \mathrm{min}$. Sampling was carried for 24 -h one day a week. The filters used were fiberglass, with size of $8 \times 10$ inch that captured TSP particles. Analysis of TSP were performed using scanning electron microscopy (SEM) JEOL Model JSM-5800LV coupled with energy dispersive spectrometer (EDS) EDAX DX prime brand, for determination of morphology and elemental composition of airborne particles. Specimens were processed by separating the collected particles from the fiberglass filters by means of submersing a $2 \mathrm{~cm} 2$ section of each filter into isopropilic alcohol within a test tube for 5 minutes. Then, an aliquot of the suspension was placed over a sample holder, and is introduced into the chamber of SEM.

The aim of the present study is to examine morphology and elemental chemical composition of single TSP particles as part of an air quality study by SEM-EDS.

The City of Hermosillo is located between latitude $20^{\circ} 01^{\prime} 00$ "and $20^{\circ} 08^{\prime} 30^{\prime \prime}$ north latitude and between the meridian $110^{\circ} 54^{\prime} 30$ "and $111^{\circ} 01^{\prime} 00^{\prime \prime}$ west longitude at an altitude of $200 \mathrm{~m}$ above sea level; is the Sonora state's capital, located to the Center-West of the coastal plain, in northwestern Mexico [2]. 
Based on EDS results, these particulate matter was primarily composed of $\mathrm{Al}$ (At\% 14.0), S (At\%14.34), W (At\%12.53), and Fe (At\%6.17). (Fig. 2). The structure of particles can be diverse and they usually present a two dimensional aspect. (Fig. 1). It is often found that such particles have a irregular morphology, outlying edges and fracture lines. The elements found in the chemical characterization are mainly related to sources from soil, incineration, combustion activities and vehicular traffic. These particles morphology and chemical composition, illustrate an abundance of natural elements within the zone. However, some of the elements presented are directly related with human activities, and are of much interest from the public health and environmental perspectives.

\section{References:}

[1] R Ramirez-Leal, H Esparza-Ponce and H Duarte-Moller, Revista Mexicana de Fisica 53 (2007), p.102.

[2] R Ramirez-Leal, M Valle-Martinez, and M Cruz-Campas, Open Journal of Air Pollution 3 (2014), p. 121.

[3] B Oroji, E Solgi and A Sadighzadeh, Aerosol and Air Quality Research 18 (2018), p. 2131.

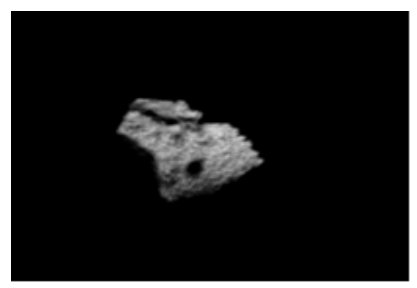

Figure 1. Micrograph of the sample in SEM-EDS. 2750x, BEI and $15 \mathrm{Kv}$

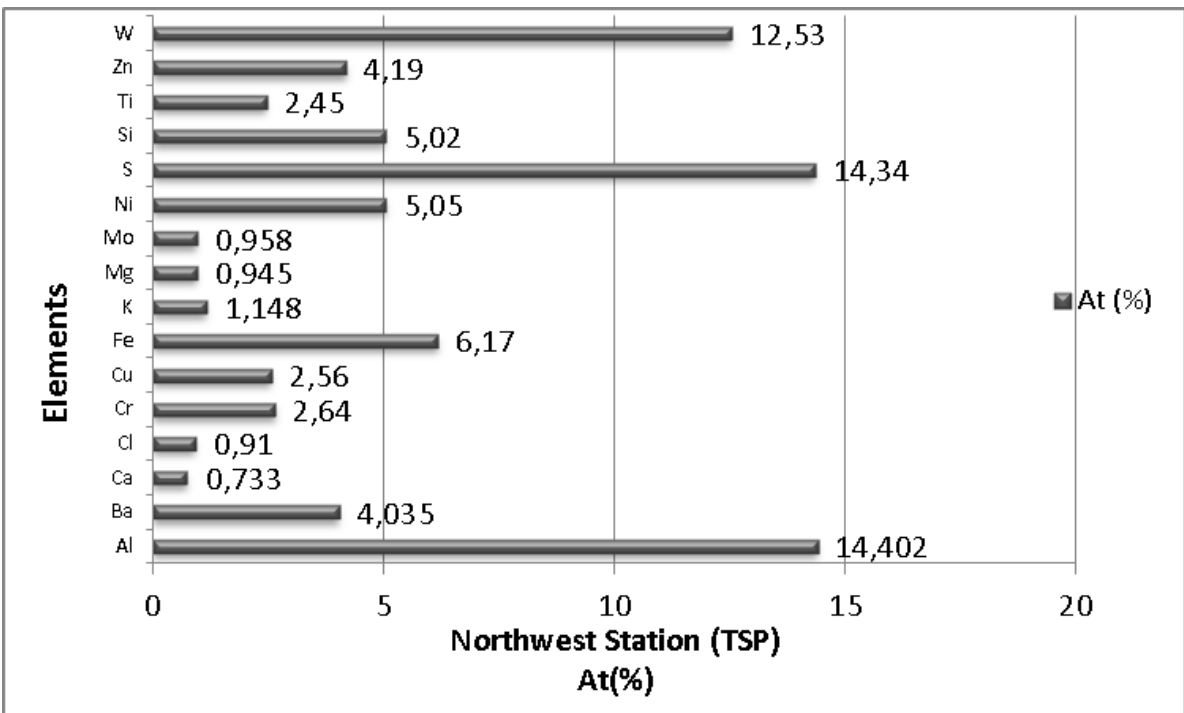

Figure 2. Majority of elemental constituents of TSP samples in northwest area (Averaged values of atomic percentage (At\%) for all filter samples). 